\title{
Local 3D Reconstruction and Augmented Reality Visualization of Free-Hand Ultrasound for Needle Biopsy Procedures
}

\author{
Ali Khamene ${ }^{1}$, Sebastian Vogt ${ }^{1}$, Fred Azar ${ }^{1}$, Tobias Sielhorst ${ }^{1}$, Frank Sauer $^{1}$, and \\ Heinrich Niemann ${ }^{2}$ \\ ${ }^{1}$ Dept. of Imaging \& Visualization, Siemens Corporate Research, \\ 755 College Road East, Princeton, NJ 08540, U.S.A. \\ \{ali.khamene, sebastian.vogt, fred.azar, tobias.sielhorst, \\ frank.sauer\}@scr.siemens.com \\ ${ }^{2}$ Dept. of Computer Science, University of Erlangen-Nürnberg, \\ Martensstr. 3, 91058 Erlangen, Germany. \\ niemanneinformatik.uni-erlangen.de
}

\begin{abstract}
We have developed a 3D ultrasound augmented reality (AR) workspace, in which the user wears a video-see-through head-mounted display to observe both 2D ultrasound (US) images and local 3D ultrasound volumes insitu, i.e. in their actual location, overlaid onto a stereoscopic video view. In this unified platform, the user has the ability to reconstruct a local threedimensional volume from a series of scanned two-dimensional US B-planes. Insitu visualization of rendered three-dimensional volumes facilitates the understanding of the object's three-dimensional structure. The user has full control over acquisition, reconstruction, and visualization of the volumetric data through an interactive, intuitive augmented reality user interface. Furthermore, the system has the ability to track a hand-held tool (e.g. biopsy needle). Graphically enhanced needle improves the perception of needle's position and orientation with respect to the lesion target in the rendered volume. In phantom experiments, our AR system has proven to effectively facilitate ultrasound guided needle biopsies. We have also verified biopsy results by reconstructing the "post-operative" volumes with the needle left in place.
\end{abstract}

\section{Introduction}

Ultrasound guidance is routinely used today in biopsy procedures. In a needle biopsy, the needle must be inserted into an anatomical target to remove a sample of tissue. $2 \mathrm{D}$ ultrasound (US) images allow the physician to locate the target and monitor the needle position in real-time. However, successful ultrasound guided needle biopsies may require considerable skill and training. 
We have shown previously that augmented reality (AR) visualization could improve intuitiveness and precision of ultrasound guided needle procedures (Sauer, Khamene et al. 2002).

We present here a new 3D ultrasound augmented reality (AR) workspace, in which the user wears a video-see-through head-mounted display to observe both $2 \mathrm{D}$ ultrasound images and local 3D ultrasound volumes in-situ, i.e. in their actual location, overlaid onto a stereoscopic video view. In this unified platform, the user has the ability to reconstruct a local three-dimensional volume from a series of scanned twodimensional US B-planes. In-situ visualization of rendered three-dimensional volumes facilitates the understanding of the object's three-dimensional structure. The user has full control over acquisition, reconstruction, and visualization of the volumetric data through an interactive, intuitive augmented reality user interface. Furthermore, the system has the ability to track a hand-held tool (e.g. biopsy needle). A graphically enhanced needle improves the perception of needle's position and orientation with respect to the lesion target in the rendered volume.

In phantom experiments, our AR system has proven to effectively facilitate ultrasound guided needle biopsies. We have also verified biopsy results by reconstructing the "post-operative" volumes with the needle left in place.

\section{Technical System Description}

Technical details of our system have been discussed in (Sauer, Khamene et al. 2001a; Sauer, Khamene et al. 2002). Here, we briefly describe the major features of the system.

\subsection{System Hardware}

We have developed a system for augmented reality visualization of medical images. The user wears a custom video-see-through head-mounted display (HMD). Two color video cameras attached to the HMD provide a stereo view of the scene. A third headmounted video camera is added for tracking. A set of optical markers ("frame") is attached to the patient's bed surrounding the desired workspace. Another set of optical markers ("cluster") is attached to the needle. The "frame" and "cluster" marker sets provide the pose of both the observer's viewpoint and the tracked needle, respectively. The system runs on a single PC and achieves real-time performance (i.e., 30 frames per second) with a latency of about $0.1 \mathrm{sec}$, generating a stable augmentation with no apparent jitter visible in the composite images. The system block diagram is shown in Fig. 1 .

\subsection{Adapting the System for 3D Ultrasound Guided Procedures}

In order to adapt the AR system for ultrasound-guided procedures, we needed to consider some modifications. Here we discuss the major system features. Additional details can be found in (Sauer, Khamene et al. 2001b) 

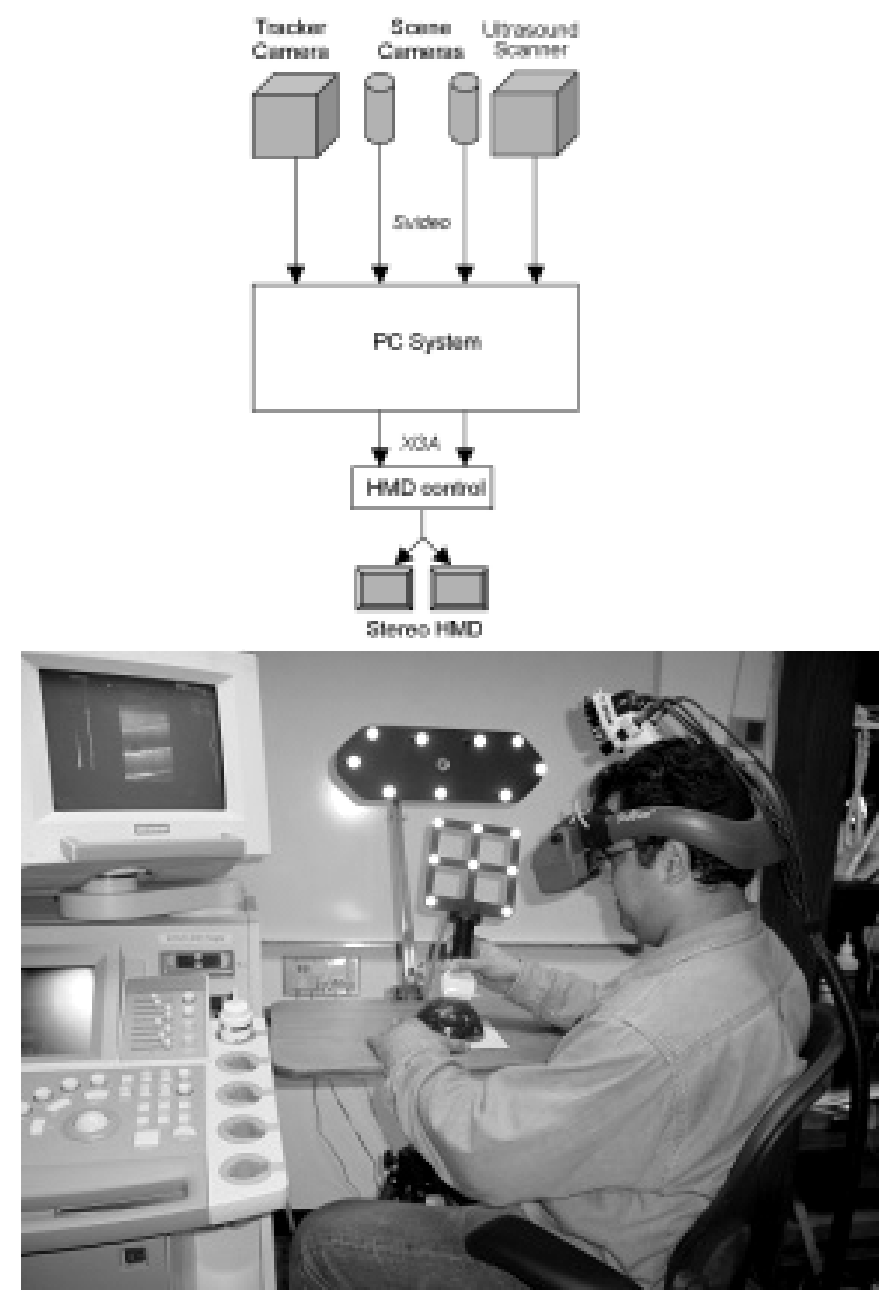

(a)

Fig. 1. (a) System block diagram, (b) Ultrasound workspace.

\subsubsection{Ultrasound Workspace}

The ultrasound workspace is shown in Fig. 1 b. For simple demonstration experiments, the user sits at a small table, which is mounted on a tripod for portability. The user wears the head-mounted display to see the stereoscopic video images of the workspace. The video images are augmented with the live images from the ultrasound scanner. The ultrasound images are registered to the pose of the ultrasound transducer. Structures in the ultrasound images are perceived at the locations of the actual physical structures.

In this setting, the "frame" marker set provides a common reference for all other tracked objects. In addition to the user's viewpoint, tracked with respect to the 
"frame" coordinate system, we need to also keep track of the position and orientation of two handheld objects, namely, the ultrasound probe, and a hand-held tool such as a biopsy needle. To do so, we need to attach two "cluster" marker sets to the desired objects.

\subsubsection{Ultrasound Calibration}

For both the AR visualization of the ultrasound images and $3 \mathrm{D}$ freehand US, the images must be correctly registered to the transducer. A number of calibration approaches for 3D free-hand ultrasound have been described in the literature (Prager, Rohling et al. 1998; Prager, Gee et al. 1999). The main difference is in the choice of the calibration object, and the tracking technology (e.g., either optical or magnetic). Since our system takes advantage of an optical tracking technology, we use a calibration object, which consists of two optical marker sets. At the top of the object, a set of disc-shaped retro-reflective markers sits on posts in a random $3 \mathrm{D}$ configuration. On the bottom, a set of five ball-shaped retro-reflective markers is arranged in a staircase fashion. These ball-shaped markers have a $12 \mathrm{~mm}$ diameter, and serve both as optical and ultrasound markers, for a precise determination of the object's 3D geometry. The coordinates of all markers are measured using an optical tracking system.

We partially submerge the calibration object into a small water tank Fig. 2a). The balls are underwater and can be detected with the ultrasound scanner; their 3D locations can be deduced by tracking the upper set of disc-shaped markers.

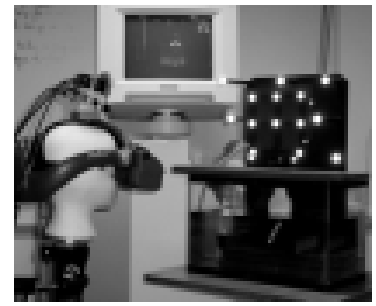

(a)

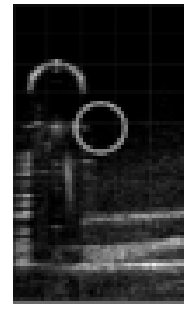

(b)

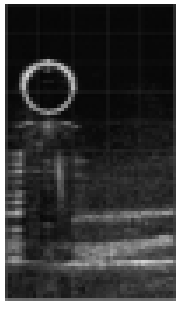

(c)

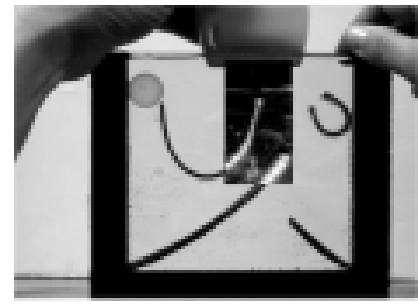

(d)

Fig. 2. (a) Ultrasound calibration setup, (b) and (c) determining the 2D location of the ball in the ultrasound image by interactive template fitting, (d) Test phantom for visualizing the ultrasound overlay accuracy.

We use the following ultrasound calibration procedure:

- We move the transducer into a position where the ultrasound plane goes right through the center of one of the balls. Such a position can be adjusted rather easily. Fig. 2 b\&c) is an example of a corresponding screen shot where the ball can be seen as a half circle. We overlay a "model" of the ball in the form of a full circle onto the screen and move it into a fitting location to estimate the $2 \mathrm{D}$ coordinates of the ball in the ultrasound image. Because of the symmetry of the ball's image, its horizontal location can be determined very accurately. The accuracy of the vertical location depends on the correct size of the template. For additional 
height cues one could construct the calibration object with small platforms under the balls that indicate the bottom of the balls in the ultrasound images.

- We repeat the procedure of "move transducer above ball - locate ball in ultrasound image" for all the balls and several image locations of each ball, always keeping track of the transducer's pose. Ideally, one would expect that all 3D ball locations collected in this way lie on a plane with respect to a coordinate system fixed at the transducer. Currently, the template matching is performed manually, but could easily be automated.

- The correspondences between the balls' 3D coordinates in the transducer system and their 2D coordinates in the ultrasound image allow one to calculate the mapping from the image to this plane in the transducer's coordinate system.

- We collect a set of 20 measurements at 4 different horizontal positions of the probe for each of the 5 balls. Based on point correspondences, we then determine the pose of the ultrasound images in the transducer coordinate system, involving 8 degrees of freedom: 3 for translation, 3 for rotation, and 2 for horizontal and vertical scaling. The results have the following statistics:

3D ball coordinates, measured by tracking of transducer and calibration object vs. determined by tracking of calibrated transducer: $1.05 \mathrm{~mm}$ RMS and $1.6 \mathrm{~mm} \max$ deviation.

Distance of 3D ball coordinates, as measured by tracking of transducer and calibration object, from plane of calibrated ultrasound transducer: $0.74 \mathrm{~mm}$ RMS and 1.3 mm max deviation.

We fabricated a phantom with visible rubber structures to demonstrate the accuracy of the AR overlay. When one observes this phantom from approximately the normal direction, refraction does not displace the internal structures in the optical image, and one can appreciate the accuracy of the ultrasound overlay (Fig. 2 $\mathrm{d}$ ).

\subsubsection{Needle Calibration}

We need to find the coordinates of the needle tip with respect to the coordinate system established by the marker cluster attached to the needle. For that purpose, the user fixes the needle tip on a point and rotates the needle around the fixed position. By tracking the cluster of markers, the system locates the stationary point, which corresponds to the tip of the needle. In real-time tracking mode, the needle pose is then computed from that of the marker cluster attached to the needle. We augment the needle with a virtual cylindrical tube representing the needle and its extrapolated trajectory. Precise needle calibration is essential to correctly augment the needle, which is required for a successful biopsy. The main assumption here is that the needle is rigidly attached to the marker cluster, and does not bend.

\section{3D Ultrasound Implementation}

In 3D freehand ultrasound, once the probe is instrumented with a set of markers for tracking, a series of two-dimensional B-planes is acquired together with location 
information. The recorded series can then be reconstructed into a regular $3 \mathrm{D}$ voxel array for visualization and analysis.

Major issues for reconstruction of 3D ultrasound are namely:

- tracking accuracy (i.e, extensively discussed in (Vogt, Khamene et al. 2002)),

- calibration of the free-hand system (i.e., explained in section 2.2.2),

- $\quad$ synchronization of the ultrasound images and positioning information, and

- $\quad$ spatial compounding of the ultrasound B-plane data into a 3D voxel array.

Synchronization of the ultrasound images with the positioning information has been discussed in (Prager, Gee et al. 1999). We tackle this problem by matching the ultrasound refresh rate to that of the video signal containing the ultrasound image (i.e., $30 \mathrm{fps}$ ). Then we synchronize the tracking camera signal to the video out of the ultrasound device. However, there could still be a constant time lag/lead between the US images and their tracked position, which can be avoided by instructing the user to "slowly" move the transducer, while the system is gathering the data.

Next, we describe the algorithm for spatial compounding of the B-plane slices, as well as an approach to visualize the $3 \mathrm{D}$ volume resulting from AR guided freehand movement of the probe.

\subsection{Reconstruction of Free-Hand Scans}

Reconstructing the tracked ultrasound B-plane slices into a regular three-dimensional array makes it possible to use conventional 3D visualization and data analysis tools (Prager, Gee et al. 1999). These tools may include any plane re-slicing, volume rendering, segmentation and registration procedures. The reconstruction step is important since the loss of image quality and introduction of artifacts have to be avoided. Reconstruction methods can be classified to two major groups, first interpolation based, and second kernel based. Either of these methods can accommodate how to deal with voxels that could have multiple entries assigned to them. Some filtering technique could effectively take advantage of multiple observations at a sample voxel and combine them to reduce SNR of the resultant compounded volume.

In this work, we use a kernel-based method since the speed of the computation is much higher than with the other option. We choose the kernel to be one dimensional with fixed length, and parallel to the normal of the ultrasound B-plane. We also use simple averaging to deal with voxels with multiple entries, mainly due to the algorithm's low computational complexity. We next explain how the augmented reality aspect of the system helps the user go through the process of gathering, reconstructing, and visualizing the data.

\subsection{Local 3D Ultrasound and AR Visualization}

Utilization of augmented reality helps in many ways. The entire process of gathering the B-plane slices and creating the $3 \mathrm{D}$ view is presented to the user in an augmented fashion, with a high level of interactivity. The procedure is illustrated below with AR 
visualization of a test object. The test object is a screwdriver, immersed in water to maximize quality of the US acquisitions Fig. 3. The process can be divided into two tasks:

- Creating a bounding box: first, the user adjusts the location and size of a bounding box through a gaze user interface, then freezes the box into the "frame" coordinate system (Fig. 3).

- 3D Scanning: There are two major modes for scanning and reconstructing the 3D volume (Fig. 3p):

o Fan motion mode: the ultrasound probe is fixed and rotated around the axis of the acoustic transducer. For most applications this method is desired since it somehow insures that tissue deformation remains constant through out the scanning.

o Translation motion mode: the ultrasound probe is moved along the normal of the ultrasound plane. It is however conceivable that tissue deformation may occur and the resulting volume may be distorted, especially on the edges, closer to the transducer.

Once the scanning mode is selected, the augmented view can guide the user on how to move the transducer and with what speed. The reason for that is to make sure, that we have minimized the number of unsampled voxels, and to uniformly space the ultrasound B-plane data into the volume.

Fig. 3 c\&d) show the screwdriver view with and without augmentation, and Fig. 目e shows the reconstructed ultrasound volume rendering of the screwdriver's tip. Figure 3 ( $\mathrm{f} \& \mathrm{~g}$ ) show augmented reality visualization of the scanned volume from human lower arm.

\section{A Needle Biopsy Experiment}

Augmented Reality (AR) visualization has the potential to make needle placement more intuitive and precise. In fact, the first medical application proposed for AR was ultrasound guided needle biopsy, which was developed at UNC in Charlottesville (State, Livingston et al. 1996). The added capability of reconstructing a 3D volume from ultrasound images and presenting it to the user in an augmented fashion goes a long way to make the whole procedure more intuitive. We demonstrated an AR based ultrasound guided needle biopsy system in (Sauer, Khamene et al. 2002), where the user was guided via optical (e.g., laser line) and virtual lines to perform biopsies.

In this paper, we demonstrate an AR based system that allows the user to reconstruct and visualize a local 3D ultrasound volume at a location of interest, and also visualize the augmented view of the tracked needle. The needle, its extrapolated trajectory, and its tip are marked by appropriate graphics primitives to enhance the perception of the user regarding the location and orientation of the needle with respect to the target of interest within the reconstructed volume. 


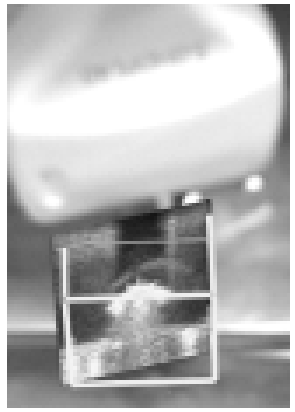

(a)

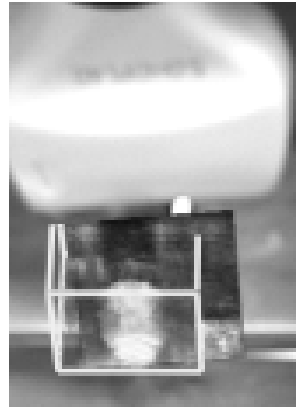

(b)

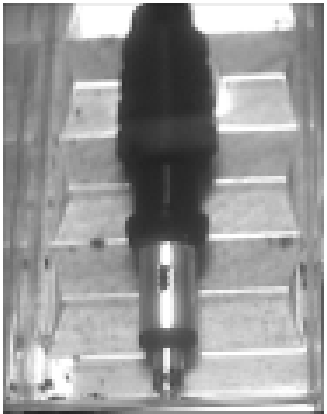

(c)

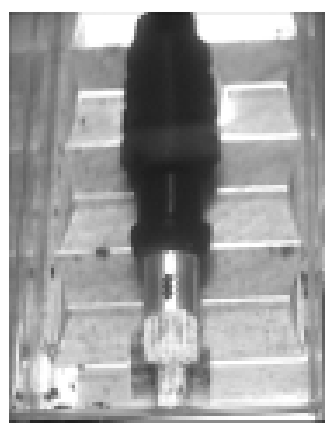

(d)

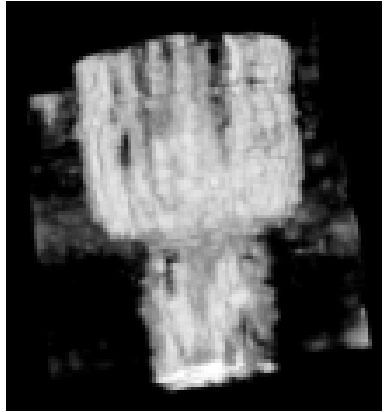

(e)

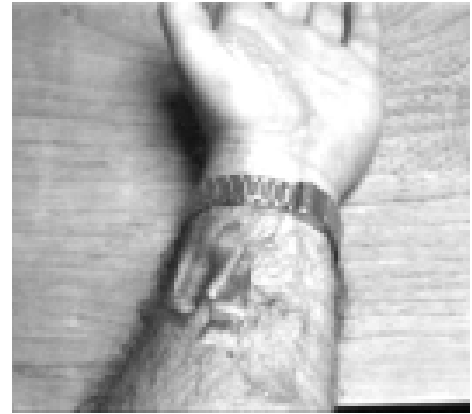

(f)

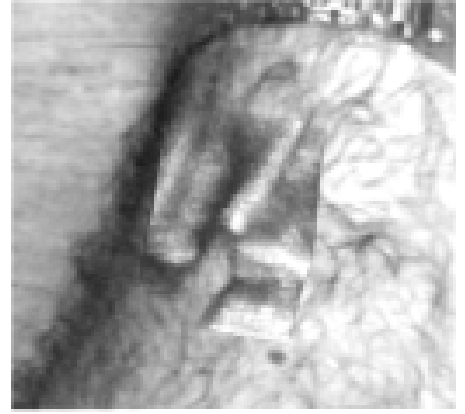

(g)

Fig. 3. Augmented reality visualization of a screwdriver test object: (a) the user adjusts the location and size of a bounding box through a gaze user interface, (b) The user reconstructs the local 3D volume inside the bounding box, (c) video view of screwdriver, (d) Augmented view of screwdriver containing the locally reconstructed ultrasound volume of the tip, (e) Reconstructed ultrasound volume rendering of the screwdriver's tip. Augmented reality visualization of human lower arm: figure (f) shows radius bone and a tendon, figure (g) is zoomed on the reconstructed volume. 


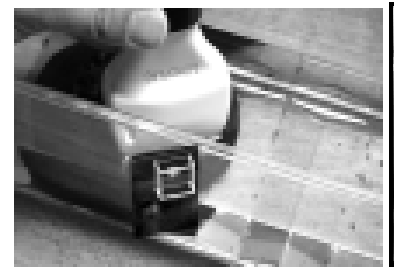

(a)

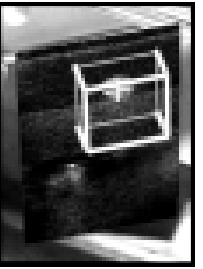

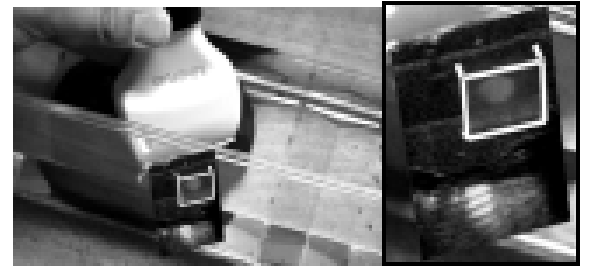

(b)

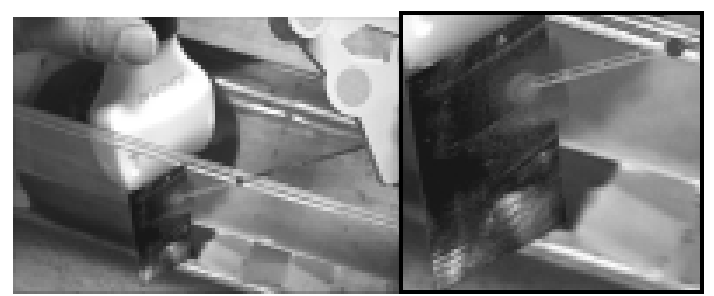

(c)

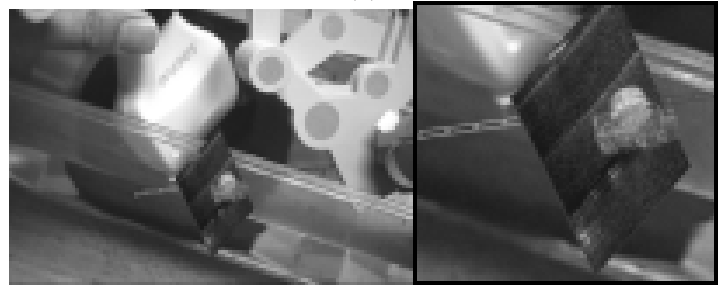

(d)

Fig. 4. Procedure for needle placement: (a) The user identifies the desired lesion, which appears on the $2 \mathrm{D}$ ultrasound scan, and places the gaze-controlled bounding box in the location of the lesion, (b) After the guided acquisition of a set of two-dimensional scans, the volume is reconstructed and visualized at the physical location where it was scanned, (c) The user places the needle on the phantom's "skin", and adjusts its orientation so that the virtual extension passes through the lesion center, (d) The user punctures the "skin", and advances the needle towards the target, until the virtual sphere representing the needle tip is placed at the center of the volume rendered lesion (note the modified transfer function of the volume rendering to get the best view of the target lesion).

\subsection{Needle Placement Procedure}

The procedure for needle placement is as follows:

- First, the user identifies the lesion location Fig. 4a), which appears on the 2D ultrasound scan, and places the gaze-controlled bounding box in the location of the lesion (as explained in section 3.2).

- The user is guided through the freehand scanning process as the volume is reconstructed and augmented onto the view of the object. (Fig. 4 p).

- The user modifies the transfer function and color palettes of the rendering in order to get a better view of the target lesion. This step is important since the 
user can utilize a number of inherent properties of volume rendering technology, such as selective visualization (Fig. 4 ).

- The user proceeds to insert the augmented needle into the lesion: the needle placement is facilitated through augmentation of the tracked needle, and its extrapolated trajectory with cylinders of different colors. Furthermore, the needle tip is enhanced with a sphere. The user punctures the breast phantom's "skin", and adjusts the needle orientation so that its virtual extension passes through the target center (Fig. 4). Finally, the user advances the needle towards the lesion, until the virtual sphere representing the needle tip appears at the center of the lesion, shown by volume rendering technology (Fig. 4 $)$.

\subsection{Experiment Results}

We performed a series of needle biopsies on an ultrasound breast biopsy phantom (Gammex RMI, Middleton, WI). Eleven test objects are embedded in the phantom. These are located on three different levels in the phantom. Test lesions include 3 water-filled cysts with $10 \mathrm{~mm}$ to $15 \mathrm{~mm}$ diameter, 4 high-density gel cysts with 7 and $10 \mathrm{~mm}$ diameter, and 4 low-density gel cysts with 7 and $10 \mathrm{~mm}$ diameter.

Following the steps outlined in section 4.1, we performed the needle biopsy procedure, with four different high-density gel lesions $(7-10 \mathrm{~mm}$ diameter). Once the needle was in place, we re-scanned the phantom and reconstructed another 3D ultrasound volume, which we called the "post-op" (for post-operative) volume.

Since the ultrasound images and volumes are inherently noisy, it is difficult to quantitatively measure the distance between the needle tip and the lesion center as we did in (Khamene, Wacker et al. 2003) for MRI guided needle biopsies. However, the rendered post-op reconstructed ultrasound volumes demonstrate the successful placement of the needle. Fig. 5 shows four post-op-scans, in which the needle and target lesions are indicated. Although the test users were novices in performing the ultrasound guided needle biopsy, they could still intuitively accomplish a successful needle placement in a short amount of time (i.e. less than 10 seconds).

\section{Summary and Conclusion}

We developed an ultrasound AR workspace where the user wears a video-seethrough head-mounted display to observe the ultrasound slices in-situ, i.e. in their actual location, overlaid onto a stereoscopic video view. The ultrasound-to-video and ultrasound-to-workspace registration is performed with a head-mounted tracker camera, which tracks markers attached to transducer and workspace. The system enables the user to specify the bounding box for the 3D volume through a gaze user interface. A local three-dimensional volume is then reconstructed, based on ultrasound slices tracked in real-time, to facilitate the understanding of the object's 3D structure. Fur- 
thermore, the biopsy needle is tracked and augmented with some graphic primitives to enhance the perception of its pose with respect to the target in the volume.
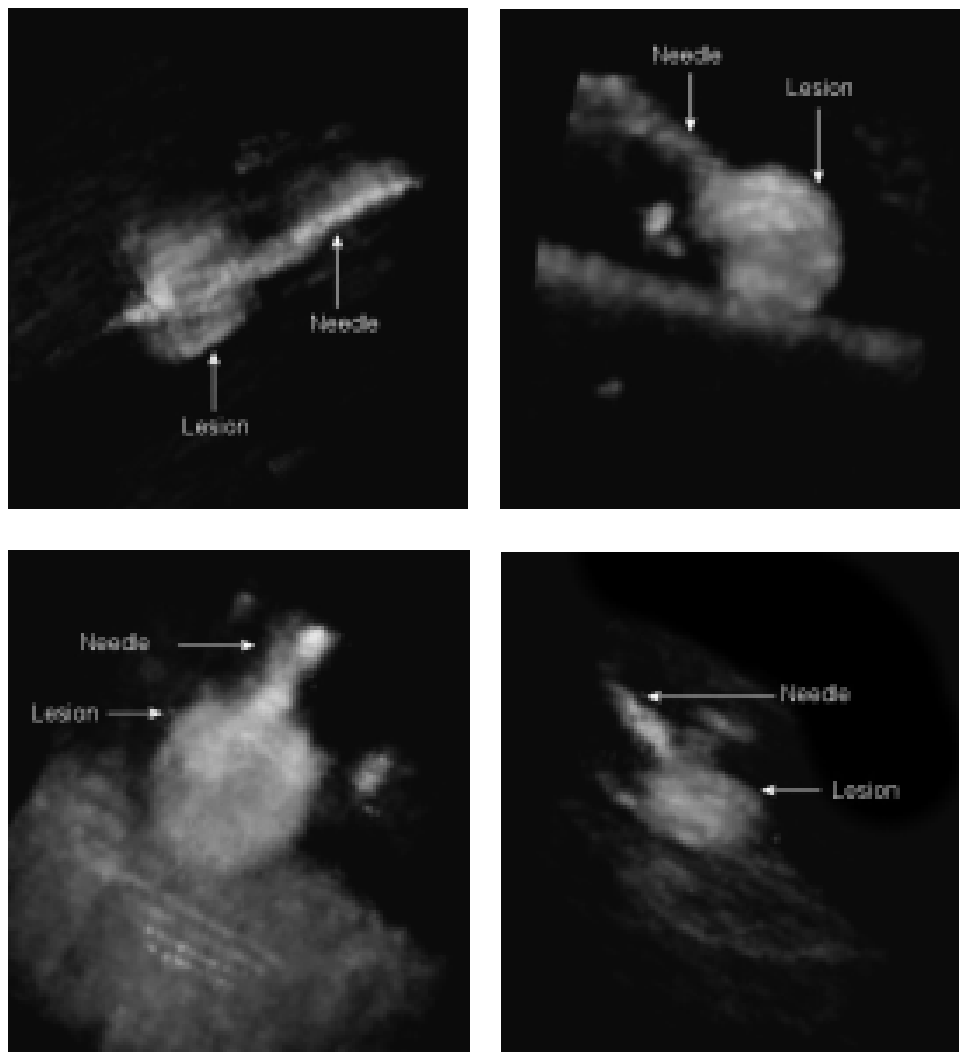

Fig. 5. 3D post-op scans of four different needle biopsy procedures, showing the needle and the 3D rendered lesions.

The novelty of this approach lies in the development of a unified AR ultrasound platform, which integrates the following processes:

- Acquisition of ultrasound B-planes from a desired location, and reconstruction of the images into a local 3D volume,

- AR visualization of the reconstructed 3D volume,

- Assisted needle placement.

In phantom experiments, our AR system has proven to effectively facilitate successful ultrasound guided needle biopsies. Our future work with the AR ultrasound system will be focused on testing the system on real cases in a clinical setting. 


\section{References}

Khamene, A., F. Wacker, et al. (2003). "An Augmented Reality System for MRI-Guided Needle Biopsies." Proceedings of Medicine Meets Virtual Reality 11, 94: 151-157.

Prager, R. W., A. H. Gee, et al. (1999). "Stradx: real-time acquisition and visualization of freehand three-dimensional ultrasound." Medical Image Analysis 3(2): 129-140.

Prager, R. W., R. N. Rohling, et al. (1998). "Rapid calibration for 3-D freehand ultrasound." Ultrasound in Medicine and Biology 24(6): 855-869.

Sauer, F., A. Khamene, et al. (2001a). "A Head-Mounted Display System for Augmented Reality Image Guidance: Towards Clinical Evaluation for iMRI-guided Neurosurgery." Proceedings of Medical Image Computing and Computer-Assisted Intervention - MICCAI'2001 (Utrecht, The Netherlands), Lecture Notes in Computer Science 2208: 707-716.

Sauer, F., A. Khamene, et al. (2001b). "Augmented Reality Visualization of Ultrasound Images: System Description, Calibration, and Features." IEEE and ACM Int. Symp. on Augmented Reality - ISAR2001 (New York, NY): 30-39.

Sauer, F., A. Khamene, et al. (2002). "An Augmented Reality System for Ultrasound Guided Needle Biopsies." Proceedings of Medicine Meets Virtual Reality 10: 455-460.

Sauer, F., F. Wenzel, et al. (2000). "Augmented workspace: designing an AR testbed." IEEE and ACM ISAR Munich, Germany October 5-6: 47-53.

State, A., M. A. Livingston, et al. (1996). "Technologies for Augmented Reality Systems: realizing Ultrasound-Guided Needle Biopsies." Proceedings of SIGGRAPH '96 in Computer Graphics Proceedings, Annual Conference Series 1996, ACM SIGGRAPH: 439-446.

Vogt, S., A. Khamene, et al. (2002). "Single Camera Tracking of Marker Clusters: MultiParameter Cluster Optimization and Experimental Verification." In Proc. IEEE \& ACM Intl. Symp. on Mixed and Augmented Reality, ISMAR: 127-136. 